\title{
Nuclear Density Dependence of In-Medium Polarization
}

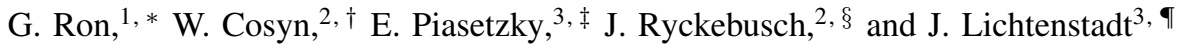 \\ ${ }^{1}$ Racah Institute of Physics, Hebrew University of Jerusalem, Givat Ram 91904, Israel \\ ${ }^{2}$ Department of Physics and Astronomy, Ghent University, Proeftuinstraat 86, B-9000 Gent, Belgium \\ ${ }^{3}$ School of Physics and Astronomy, Tel Aviv University, Tel Aviv 69978, Israel
}

(Dated: January 25, 2013)

\begin{abstract}
It is shown that polarization transfer measurements $\left(\vec{e}, e^{\prime} \vec{p}\right)$ on a specific target nucleus can provide constraints on the ratio of the in-medium electric to magnetic form factor. Thereby one exploits the fact that proton knockout from single-particle levels exhibit a specific sensitivity to the effective nuclear density. It is shown that in ${ }^{12} \mathrm{C}$ the effective nuclear density for $s$-shell knockout is about twice as high as for $p$-shell knockout. With current model predictions for the in-medium form factors, one obtains measurable modifications of the order of $5 \%$ in the ratios of the double polarization observables between those single-particle levels.
\end{abstract}

PACS numbers: 13.40.Gp,13.88.+e,25.30.Dh,11.80.-m

Nuclei are well described as ensembles of protons and neutrons held together by a strong mutual force. The nucleons are complex entities and the question of whether their internal structure is changed while they are embedded in nuclei has been a long-standing question in nuclear physics which remains unsettled [1].

The polarization transfer measured in the $p\left(\vec{e}, e^{\prime} \vec{p}\right)$ reaction is a direct measure of the ratio of the proton elastic electric to magnetic form factor (FF) ratio at some value of the fourmomentum transfer $Q^{2}$ :

$$
\frac{P_{x}}{P_{z}}=-\frac{2 M_{p}}{\left(E+E^{\prime}\right) \tan (\theta / 2)} \frac{G_{E}^{P}\left(Q^{2}\right)}{G_{M}^{P}\left(Q^{2}\right)},
$$

where $P_{x}\left(P_{z}\right)$ is the transverse (longitudinal) polarization transfer, $E\left(E^{\prime}\right)$ is the incident (scattered) electron energy, $\theta$ is the electron scattering angle, and $M_{p}$ is the proton mass (see [2] for details).

When exclusive $\left(\vec{e}, e^{\prime} \vec{p}\right)$ measurements are performed on a nuclear target, the polarization transfer observables are sensitive to the modifications of the form factors of the embedded nucleons, which we denote by $\frac{G_{E}^{*}}{G_{M}^{*}}[3-5]$. The double polarization ratios

$$
\left(\frac{\left(P_{x} / P_{z}\right)_{A}}{\left(P_{x} / P_{z}\right)_{H}}\right)
$$

taken between a knockout nucleon from a nucleus $A$ and a free nucleon, are only moderately sensitive to many-body effects like meson-exchange currents (MEC), isobar currents (IC), and final-state interactions (FSI) [6-8]. Small changes to the measured observables in nuclei due to these many-body effects are possible. Distinguishing between the latter and the in-medium nucleon structure modification is possible only using theoretical calculations. The challenge is to observe

\footnotetext{
*Electronic address: gron@phys.huji.ac.il

${ }^{\dagger}$ Electronic address: wim.cosyn@ugent.be

¥Electronic address: eip@tauphy.tau.ac.il

$\S$ Electronic address: jan.ryckebusch@ugent.be

ฯElectronic address: jech@tauphy.tau.ac.il
}

(or, exclude) deviations which are outside the theoretical and experimental uncertainties that can be used as evidence for changes in the bound nucleon form factor compared to that of a free one.

The combination of high intensity, high polarization, continuous electron beams, and high precision spectrometers with focal plane polarimeters allows a measurement of the ratio of polarization observables to a level of $1-2 \%$ [5, 9]. With such measurements the theoretical uncertainties are the limiting factor.

High-precision experiments and calculations, designed to look for differences between the in-medium polarizations and the free values, compared polarization observables measured in quasi-elastic scattering off nuclear targets to these obtained for hydrogen [5]. We discuss here the possibility to identify in-medium effects and study their local nuclear density dependence by comparing quasi-elastic proton removal from the $s$ shell and $p$-shell in ${ }^{12} \mathrm{C}$. As we show below, in these cases the local nuclear density is dramatically different.

Obtaining consistent results for medium modification if one compares $s$-shell and $p$-shell knockout protons in the ${ }^{12} \mathrm{C}\left(\vec{e}, e^{\prime} \vec{p}\right){ }^{11} \mathrm{~B}$ reaction, or if one compares the quasi-elastic scattering to that off a free proton, is a strong support that can reduce the theoretical uncertainty of the magnitude of the medium modifications. Moreover, one expects the medium modification to depend on the local nuclear density and/or the bound nucleon momentum/virtuality. Measurements that can map the effects as a function of these two variables may reveal the nature of the medium modifications.

The missing momentum $p_{m}$ corresponds to the initial momentum of the struck nucleon in plane-wave kinematics. In the deuteron, due to the low nuclear density, the expected effect of medium modifications at low missing momenta is too small to be detected unambiguously [10-13]. New measurements for high missing momentum are still unpublished [14]. Several polarization-transfer proton-knockout experiments have been performed on ${ }^{4} \mathrm{He}$, both at the MAMI facility [15] and at Jefferson Lab (JLab) [5, 16]. The doubleratio of the in-plane polarization components in ${ }^{4} \mathrm{He}$ and a free 
proton,

$$
\left(\frac{\left(P_{x} / P_{z}\right)_{H e}}{\left(P_{x} / P_{z}\right)_{H}}\right)
$$

which reflects the changes in the corresponding ratio of the electric and magnetic form factors, does not agree with stateof-the-art Distorted Wave Impulse Approximation (DWIA) calculations [5] using free nucleon form factors, but can be well described by including effects of medium modified form factors [17,-22]. However, it has recently been shown [23] that including strong effects from charge-exchange FSI can also explain the observed double-ratio of Eq. (3).

The induced proton polarization in the ${ }^{12} \mathrm{C}\left(e, e^{\prime} \vec{p}\right)$ reaction has been reported by Woo [24] at quasi-elastic kinematics and MAMI energies, covering a missing momentum range of 0 $250 \mathrm{MeV} / \mathrm{c}$. Polarization transfer measurements on ${ }^{16} \mathrm{O}$ were carried out at JLab [25]. Transverse and longitudinal polarization components were measured in quasi-elastic perpendicular kinematics at $Q^{2}=0.8(\mathrm{GeV} / \mathrm{c})^{2}$. The relatively large uncertainties on both the measurements and the calculations did not allow identification of deviations due to medium effects.

In this work we propose that the current state-of-the-art of calculations and measurements allows observing possible medium effects that are associated with local nuclear density. This can be done by comparing quasi-elastic $s$-shell and $p$-shell removal of protons from ${ }^{12} \mathrm{C}$ rather than comparing quasi-elastic to elastic scattering off Hydrogen.

We start by briefly presenting the Relativistic Multiple Scattering Glauber Approximation (RMSGA). We then discuss the local nuclear density difference between $s$ - and $p$-shell protons in ${ }^{12} \mathrm{C}$ and present a few model calculations that estimate the magnitude of the expected effect of medium modifications on double polarization observables. We follow by proposing kinematics that can be accessed at the MAMI/A1 [26, 27] facility, estimate the expected uncertainty, and compare it to the size of the expected density dependent in-medium effect. Finally we discuss the possible conclusions that can be drawn given the theoretical and experimental limitations.

The Relativistic Multiple-Scattering Glauber Approximation (RMSGA) [28] is the theoretical framework used in this work. It is a parameter-free model that was used to describe well cross sections, nuclear transparencies and other observables in a large variety of electron and hadron induced exclusive reactions in kinematical conditions close to the case we discuss here [21, 29, 30]. The RMSGA provides an unfactorized approach to the $\left(e, e^{\prime} p\right)$ reaction. In contrast to factorized models which write the cross section in an electronproton part times a FSI-corrected nuclear-structure part, the RMSGA computes the cross sections starting from the amplitudes. In the RMSGA the reaction amplitudes can be factorized in a part that describes the wave function of the proton in the nuclear ground state, times an off-shell current operator for the electron-proton scattering, times an attenuation factor that accounts for the FSI of the emerging proton. The eikonal Glauber FSI phase is a scalar in spin-space, hence the FSI do not contain any spin effects. The proton in the nuclear ground state is described by a single-particle bound state wave func- tion obtained from the Serot-Walecka model [31]. To describe the polarization observable in the polarized electron scattering off the bound proton, the off-shell cross section $\mathrm{CC} 2$ was used [32]. FSI were calculated using a relativistic extension of the Glauber approximation. In the computation of the FSI, the local nuclear density obtained from the independent-particle wave function was corrected for the short-range correlations (SRC) assuming Jastrow correlation function [33].

The effective density $\langle\rho(r)\rangle$ for both the $s$-shell and the $p$-shell proton in quasi-elastic proton knockout from ${ }^{12} \mathrm{C}$ are shown in Fig. 1 as a function of missing momentum. These densities are obtained with mean-field single-particle wave functions. We observe the effective density probed in proton knockout from the $s$-shell is about $0.1 \mathrm{fm}^{-3}$ and rises slightly with increasing missing momentum $p_{m}$. This is more than double the density probed in knockout from the $p$-shell, which is around $0.04 \mathrm{fm}^{-3}$. Also shown in the figure is $\delta(r)$ which is the calculated contribution from an infinitesimal density interval $[r, r+d r]$ to the cross section for a quasifree ${ }^{12} \mathrm{C}\left(e, e^{\prime} p\right)$ process and accounts for the effect of FSI and SRC therein. For a more detailed introduction on the quantity $\delta(r)$ we refer to Refs. [29, 34]. The FSI cause the largest contributions to the cross section to stem from the peripheral regions of the proton densities. These FSI effects are strongest for the highdensity regions of the nucleus and thus affect the $s$-shell more than the $p$-shell.

To estimate the size of the in-medium modification we use two models with density-dependent medium-modified elastic form factors for the description of a bound proton. Fig. 2 shows the nuclear density dependence of the proton EM form factors at $Q^{2}=0.4(\mathrm{GeV} / \mathrm{c})^{2}$ described by the two models. In the Chiral Quark Soliton (CQS) model [19, 35] the sea quarks are almost completely unaffected, whereas the valence quarks yield significant modifications of the form factors in the nuclear environment. The model yields a decrease of the electric form factor of about $5 \%$ at nuclear saturation density $\left(\sim 0.16 \mathrm{fm}^{-3}\right)$, while the modification of the magnetic form factor is smaller, around 1-2\%. In the Quark Meson Coupling (QMC) model [17, 36] the form factors are found to be increasingly modified as the nuclear density increases. For example, at saturation nuclear density, the nucleon electric form factor is, reduced by approximately $7 \%$, similar to the CQS model. The magnetic form factor increases by about the same amount, which is quite different from the CQS value.

These QMC and CQS model calculations contained in Fig. 2 do not intend to yield precise predictions for the proposed ${ }^{12} \mathrm{C}\left(e, e^{\prime} \vec{p}\right)$ measurement, neither to test/select the most appropriate model. These calculations point to the possible size of the effect we expect to see from scattering off the tightly bound $s$-shell proton relative to the less bound $p$-shell proton. See Fig. 3 for an estimate of the difference between the two shell removals for realistic measurement conditions discussed below.

The suggested measurements can be performed using the MAMI/A1 beam line and spectrometers [26, 27]. A $20 \mu \mathrm{A}$, $600 \mathrm{MeV}$, electron beam can be used to bombard a solid thin carbon target. Two high resolution, small solid angle, spectrometers will be used to detect the scattered electron and pro- 

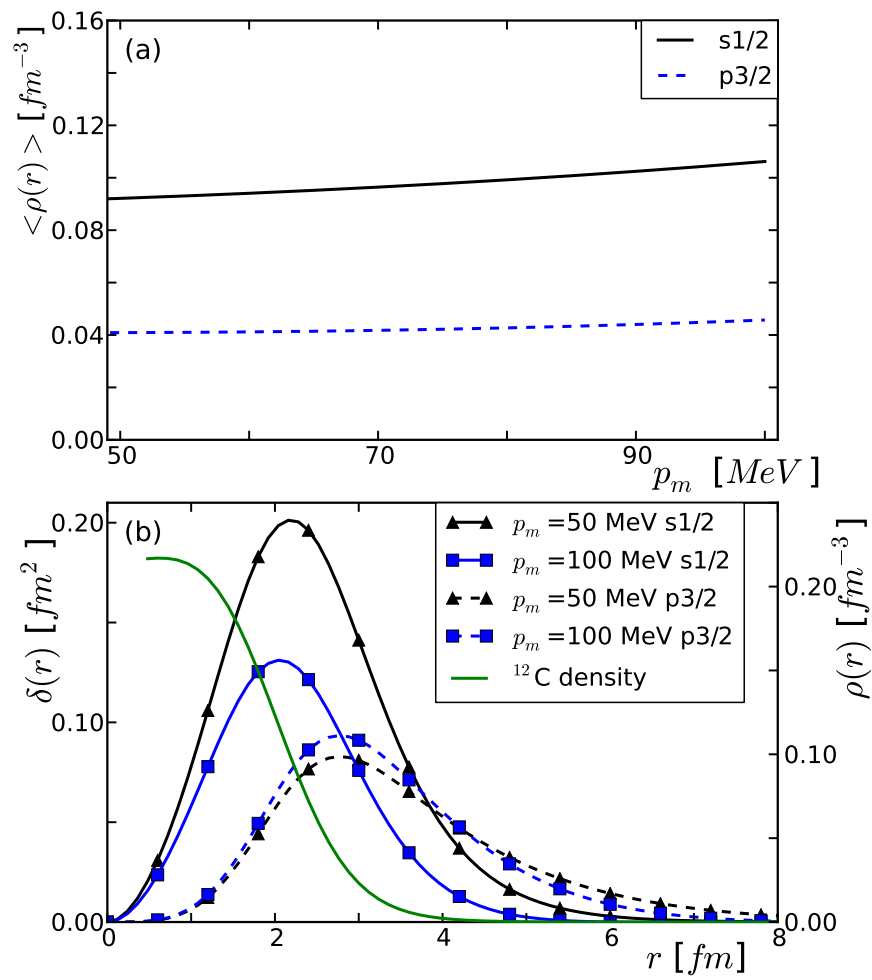

FIG. 1: (Color online) (a) Effective densities for protons removed from the $s$-shell and $p$-shell at $Q^{2}=0.4(\mathrm{GeV} / \mathrm{c})^{2}$ as a function of missing momentum. (b) $\delta(r)$ for $s$-shell (full) and $p$-shell (dashed) removal for a missing momentum of 50 (black curves) and 100 (blue curves) $\mathrm{MeV}$. The ${ }^{12} \mathrm{C}$ density is also plotted (green curve) as a reference (scale on the right-side $y$-axis).

ton. The MAMI/A1 spectrometers have a scattering angle acceptance of approximately \pm 4 degrees, and a momentum acceptance of $20-25 \%$. The spectrometer used to detect the proton is equipped with a focal plane polarimeter (FPP) that is used to measure the polarization of the recoil proton. The momentum resolution achievable by this setup allow reconstructing the missing mass and clearly identifying the $s$ - and $p$ - removal protons, which are separated by more than $2 \mathrm{MeV}$.

The proposed kinematics are, $Q^{2}=0.4(\mathrm{GeV} / \mathrm{c})^{2}$, a beam energy of $600 \mathrm{MeV}$, which gives a scattered electron energy of $E^{\prime}=384 \mathrm{MeV}$, and a scattering angle of 82.4 (34.7) degree for the electron (proton). This setup covers a missing momentum range of approximately $0 \pm 100 \mathrm{MeV} / \mathrm{c}$. At these kinematics the cross section is large enough so that the data rate is limited by the Data Acquisition System. The analyzing power of the FPP, and the spin precession angle of the proton in the spectrometer magnetic field are such that within a reasonable amount of beam time ( $\sim 2$ weeks) enough statistics can be collected to ensure that the statistical uncertainties are smaller than both the systematic and theoretical uncertainties. The expected systematic uncertainties are dominated by the spin precession of the proton in the magnetic field of the spectrometer, requiring an accurate reconstruction of the proton trajectory in the magnetic field, as well as knowledge of the field map. Comparison of the measured polariza-

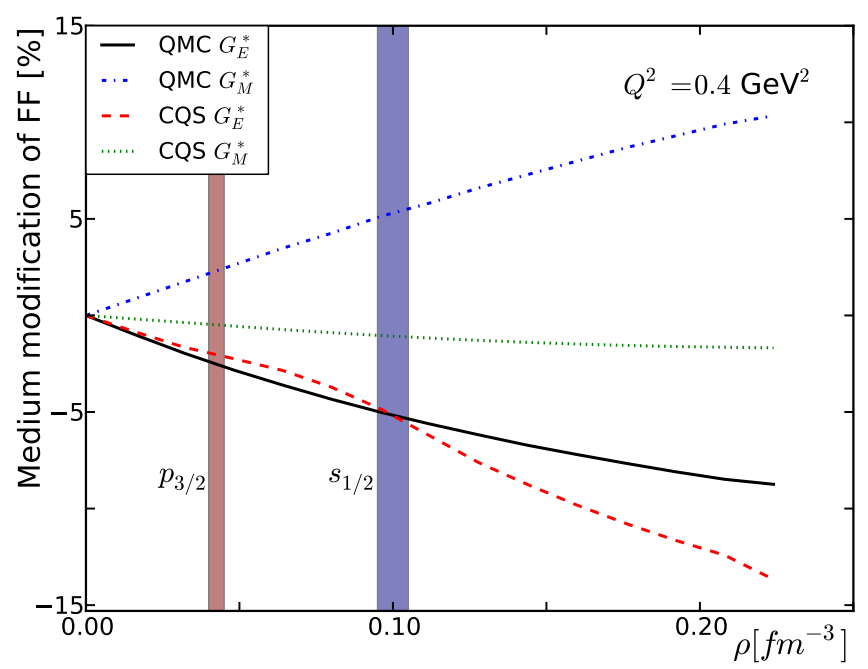

FIG. 2: (Color online) The nuclear density dependence of the proton EM form factors from the QMC and CQS models as a function of nuclear density at $Q^{2}=0.4(\mathrm{GeV} / \mathrm{c})^{2}$. The shaded bands show the effective nuclear densities for the two proton shells probed in the ${ }^{12} \mathrm{C}\left(e, e^{\prime} p\right)$ reaction at these kinematics.

tion components with the well known results for a free proton at the same $\mathrm{Q}^{2}$ can be used to test the systematic uncertainties. The false asymmetries are removed by using straightthrough runs, where the carbon analyzer is removed, resulting in straight tracks throughout the polarimeter chambers. We estimate based on previous results [5, 9, 15], a conservative systematic uncertainty of $2 \%$ in the polarization ratio. Note, however, that this estimate is for the full acceptance of the spectrometer. The comparison of the polarization ratios for sshell and p-shell protons can be performed for individual parts of the focal plane and then combined. This procedure reduces the variation of the trajectories through the magnetic field, and its contribution to the systematic uncertainty.

Fig. 3 shows the predicted ratio of $s-$ and $p-$ shell removal calculations with in-medium modification to the modification free ratio. The CQS and QMC models discussed above were used to describe the in-medium case, the modificationfree ratio was calculated with free proton form factors (i.e., no medium modification). All predictions use the RMSGA framework. The ratio is shown as a function of the $\left(e, e^{\prime} p\right)$ missing momentum $p_{m}$ and integrated over the acceptance of the MAMI/A1 spectrometers as listed above. So Fig. 3 is our estimate of the result of the proposed measurement.

In Fig. 3 super double ratios substantially different from unity are an indication of in-medium modification. As can be deduced from Fig. 3 the expected effect is about $5 \%$. With four $p_{m}$ bins (measured simultaneously) each measured with $1-2 \%$ uncertainty, the deviation from unity can be determined with very high certainty.

To summarize, we propose a measurement of the polarization transfer components of the $s$-shell and $p$-shell knockout protons in the ${ }^{12} \mathrm{C}\left(\vec{e}, e^{\prime} \vec{p}\right){ }^{11} \mathrm{~B}$ reaction using the MAMI/A1 spectrometers. A free nucleon placed in the strong field of the nucleus can have its structure modified. Proton recoil polar- 


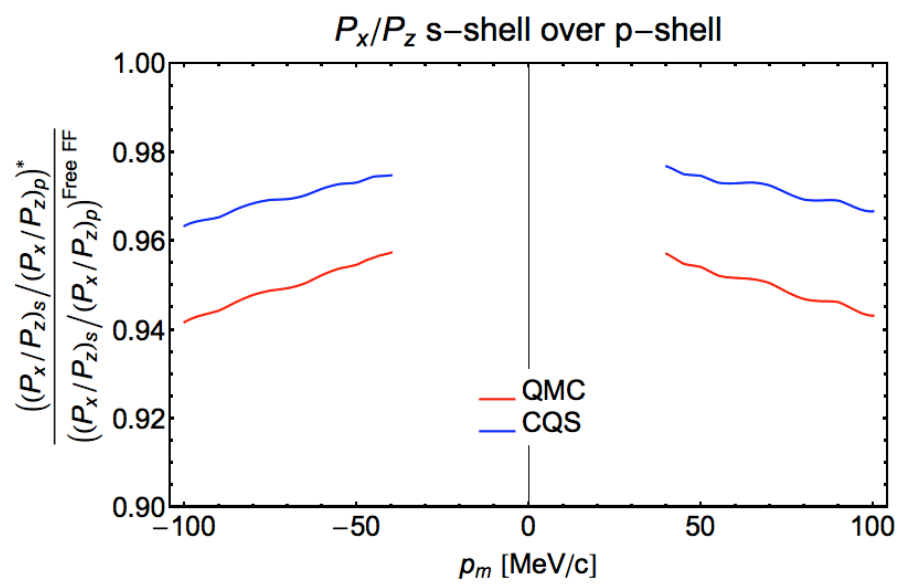

FIG. 3: (Color online) The ratio of the expected in-medium modification effect in the $s$ - and $p$-shell removals. See text for details.

ization, measured in the quasi-elastic ${ }^{4} \mathrm{He}\left(\vec{e}, e^{\prime} \vec{p}\right){ }^{3} \mathrm{H}$ reaction, with unprecedented precision, were found to be different from those of the ${ }^{1} \mathrm{H}\left(\vec{e}, e^{\prime} \vec{p}\right)$ reaction, and studied as a function of proton virtuality. Large virtuality was also claimed to be the main cause of the EMC effect [37, 38]. Another possible source of in-medium modification of the nucleon properties is the local nuclear density probed in the reaction. Even at low missing momentum one can expect off-shell behavior which most probably depends on the nuclear density. In ${ }^{12} \mathrm{C}$, with high precision measurements, we can identify proton removal from the $p$-shell and $s$-shell and study possible changes between these two cases, where the local density is more than a factor of two different. In both the CQS and QMC model our calculations show modification effects around $5 \%$ for the double ratio $\left(P_{x} / P_{z}\right)_{s} /\left(P_{x} / P_{z}\right)_{p}$ at the proposed kinematics.

The authors wish to thank Harald Merkel and Micheal Distler for fruitful discussions about the Mainz MAMI/AQ1 facility. This work is supported by the German Israeli Foundation, Grant No. 2257-2141.14/2010, the Israel Science Foundation, Grant No. 138/11, and the Research Foundation Flanders. Computational resources (Stevin Supercomputer Infrastructure) and services used in this work were provided by Ghent University, the Hercules Foundation, and the Flemish Government department EWI.
[1] A. Bracco, P. Chomaz, J. Gaardhøje, P.-H. Heenen, G. Rosner, E. Widmann, and G.-E. Körner, NuPECC Long Range Plan 2010: Perspectives of Nuclear Physics in Europe (2010), URL http://www.nupecc.org/

[2] A. I. Akhiezer and M. P. Rekalo, Sov. J. Part. Nucl. 4, 277 (1974).

[3] I. Cloet, G. A. Miller, E. Piasetzky, and G. Ron, Phys. Rev. Lett. 103, 082301 (2009).

[4] S. Strauch, S. Malace, and M. Paolone (2009).

[5] M. Paolone, S. P. Malace, S. Strauch, I. Albayrak, J. Arrington, B. L. Berman, E. J. Brash, B. Briscoe, A. Camsonne, J.-P. Chen, et al. (E03-104 Collaboration), Phys. Rev. Lett. 105, 072001 (2010).

[6] J.-M. Laget, Nucl. Phys. A579, 333 (1994).

[7] J. J. Kelly, Phys. Rev. C59, 3256 (1999).

[8] J. Ryckebusch, D. Debruyne, W. Van Nespen, and S. Janssen, Phys. Rev. C60, 034604 (1999).

[9] X. Zhan, K. Allada, D. Armstrong, J. Arrington, W. Bertozzi, W. Boeglin, J.-P. Chen, K. Chirapatpimol, S. Choi, E. Chudakov, et al., Phys. Lett. B705, 59 (2011).

[10] D. Eyl, A. Frey, H. Andresen, J. Annand, K. Aulenbacher, J. Becker, J. Blume-Werry, T. Dombo, P. Drescher, H. Fischer, et al., Z. Phys. A352, 211 (1995).

[11] B. D. Milbrath, J. I. McIntyre, C. S. Armstrong, D. H. Barkhuff, W. Bertozzi, J. P. Chen, D. Dale, G. Dodson, K. A. Dow, M. B. Epstein, et al. (Bates FPP Collaboration), Phys. Rev. Lett. 80, 452 (1998).

[12] D. Barkhuff, C. Armstrong, W. Bertozzi, J. Chen, D. Dale, G. Dodson, K. Dow, M. Epstein, M. Farkhondeh, J. Finn, et al., Phys. Lett. B470, 39 (1999).

[13] B. Hu, M. K. Jones, P. E. Ulmer, H. Arenhövel, O. K. Baker, W. Bertozzi, E. J. Brash, J. Calarco, J.-P. Chen, E. Chudakov, et al., Phys. Rev. C 73, 064004 (2006).

[14] I. Yaron et al., To be submitted (2013).
[15] S. Dieterich, P. Bartsch, D. Baumann, J. Bermuth, K. Bohinc, R. Bhm, D. Bosnar, S. Derber, M. Ding, M. Distler, et al., Phys. Lett. B500, 47 (2001).

[16] S. Strauch, S. Dieterich, K. A. Aniol, J. R. M. Annand, O. K. Baker, W. Bertozzi, M. Boswell, E. J. Brash, Z. Chai, J.-P. Chen, et al., Phys. Rev. Lett. 91, 052301 (2003).

[17] D.-H. Lu, A. W. Thomas, K. Tsushima, A. G. Williams, and K. Saito, Phys. Lett. B417, 217 (1998).

[18] J. R. Smith and G. A. Miller, Phys. Rev. Lett. 91, 212301 (2003).

[19] J. R. Smith and G. A. Miller, Phys. Rev. C70, 065205 (2004).

[20] T. Horikawa and W. Bentz, Nucl. Phys. A762, 102 (2005).

[21] P. Lava, J. Ryckebusch, and B. Van Overmeire, Prog. Part. Nucl. Phys. 55, 437 (2005).

[22] I. Cloet, W. Bentz, and A. W. Thomas, Phys. Lett. B621, 246 (2005).

[23] R. Schiavilla, O. Benhar, A. Kievsky, L. E. Marcucci, and M. Viviani, Phys. Rev. Lett. 94, 072303 (2005).

[24] R. J. Woo, D. H. Barkhuff, W. Bertozzi, J. P. Chen, D. Dale, G. Dodson, K. A. Dow, M. B. Epstein, M. Farkhondeh, J. M. Finn, et al. (Bates FPP Collaboration), Phys. Rev. Lett. 80, 456 (1998).

[25] S. Malov, K. Wijesooriya, F. T. Baker, L. Bimbot, E. J. Brash, C. C. Chang, J. M. Finn, K. G. Fissum, J. Gao, R. Gilman, et al., Phys. Rev. C 62, 057302 (2000).

[26] K. Blomqvist, W. Boeglin, R. Bhm, M. Distler, R. Edelhoff, J. Friedrich, R. Geiges, P. Jennewein, M. Kahrau, M. Korn, et al., Nuclear Instruments and Methods in Physics Research Section A: Accelerators, Spectrometers, Detectors and Associated Equipment 403, 263 (1998).

[27] T. Pospischil, P. Bartsch, D. Baumann, R. Bhm, K. Bohinc, N. Clawiter, M. Ding, S. Derber, M. Distler, D. Elsner, et al., Nuclear Instruments and Methods in Physics Research Section A: Accelerators, Spectrometers, Detectors and Associated 
Equipment 483, 713 (2002).

[28] J. Ryckebusch, D. Debruyne, P. Lava, S. Janssen, B. Van Overmeire, and T. Van Cauteren, Nucl. Phys. A728, 226 (2003).

[29] J. Ryckebusch, W. Cosyn, and M. Vanhalst, Phys. Rev. C 83, 054601 (2011).

[30] W. Cosyn and J. Ryckebusch, Few Body Syst. 49, 77 (2011).

[31] R. Furnstahl, B. D. Serot, and H.-B. Tang, Nucl. Phys. A615, 441 (1997).

[32] T. De Forest, Nucl. Phys. A392, 232 (1983).

[33] W. Cosyn, M. C. Martinez, and J. Ryckebusch, Phys. Rev. C 77, 034602 (2008).
[34] W. Cosyn and J. Ryckebusch, Phys. Rev. C80, 011602 (2009).

[35] C. Christov, A. Gorski, K. Goeke, and P. Pobylitsa, Nucl. Phys. A592, 513 (1995).

[36] D.-H. Lu, K. Tsushima, A. W. Thomas, A. G. Williams, and K. Saito, Phys. Rev. C60, 068201 (1999).

[37] C. Ciofi degli Atti, L. Frankfurt, L. Kaptari, and M. Strikman, Phys. Rev. C76, 055206 (2007).

[38] L. B. Weinstein, E. Piasetzky, D. W. Higinbotham, J. Gomez, O. Hen, and R. Shneor, Phys. Rev. Lett. 106, 052301 (2011). 